\title{
COMPARAÇÃO ENTRE AS REGIÕES VITÍCOLAS DE SÃO JOAQUIM - SC, BRASIL E SAN MICHELE ALL'ADIGE - TN, ITÁLIA ${ }^{1}$
}

\author{
ALBERTO FONTANELLA BRIGHENTI ${ }^{2}$, LUCIANE ISABEL MALINOVSKI ${ }^{3}$, \\ MARCO STEFANINI ${ }^{4}$, HAMILTON JUSTINO VIEIRA ${ }^{5}$, APARECIDO LIMA DA SILVA ${ }^{6}$
}

RESUMO - A fenologia da videira, a produtividade das plantas e a qualidade da uva apresentam forte relação com os fatores ambientais. O objetivo deste trabalho foi comparar as regiões vitícolas de altitude elevada de Santa Catarina com a região de San Michele All'Adige localizada na Itália. Para os estudos, utilizou-se dos dados da Estação Experimental de São Joaquim - EPAGRI (28 $16^{\prime} 30,08^{\prime \prime}$, $49^{\circ} 56^{\prime} 09,34^{\prime \prime}$ ', altitude 1.400 m), localizada em São Joaquim e do Istituto Agrario di San Michele All'Adige - Fondazione Edmund Mach ( $46^{\circ} 11^{\prime} 41,46^{\prime} \mathrm{N}, 11^{\circ} 08^{\prime} 04,41^{\prime}$ 'L, altitude $223 \mathrm{~m}$ ), localizado na Província de Trento. A variedade avaliada foi a Rebo, para a qual foram determinados os principais estádios fenológicos e calculada a exigência térmica através dos graus-dia, temperaturas média, máxima, mínima, amplitude, precipitação, umidade relativa do ar, insolação e radiação solar global. No momento da colheita, foi avaliado o desempenho agronômico da variedade Rebo. Em São Joaquim, o ciclo da variedade Rebo é mais longo, o acúmulo térmico é menor, a precipitação pluviométrica e a radiação solar global acumulada são maiores do que em San Michele All'Adige. As temperaturas mais baixas e a menor insolação durante a floração-mudança de cor das bagas são os principais fatores climáticos relacionados com as menores produtividades das plantas. Em São Joaquim, as baixas temperaturas e as elevadas taxas de radiação solar global na maturação contribuem de maneira positiva na formação dos compostos fenólicos, mas dificultam a degradação dos ácidos da variedade Rebo. Termos para indexação: Vitis vinifera L., radiação solar global, graus-dia, fenologia.

\section{COMPARISON BETWEEN THE WINE PRODUCING REGIONS OF SÃO JOAQUIM - SC, BRAZIL AND SAN MICHELE ALL'ADIGE - TN, ITALY}

\begin{abstract}
The grapevine phenology, plant yield and grape quality have a strong relationship with environmental factors. The objective of this study was to compare the wine producing regions of the State of Santa Catarina in Brazil and the region of San Michele All'Adige located in Italy. The data used in this study came from São Joaquim Experimental Station - EPAGRI (28 $16^{\prime} 30,08^{\prime \prime}$, $49^{\circ} 56^{\prime} 09,34^{\prime \prime}$, altitude $1,400 \mathrm{~m}$ ), located in São Joaquim and from Istituto Agrario di San Michele All'Adige - Fondazione Edmund Mach ( $46^{\circ} 11^{\prime} 41,46^{~ ' N}, 11^{\circ} 08^{\prime} 04,41^{\prime}$ "E, altitude $\left.223 \mathrm{~m}\right)$, located in the Province of Trento. The evaluated variety was Rebo, the main phenological stages were recorded and thermal requirement was calculated through the growing degree-days. The climatic parameters evaluated were, maximum, mean and minimum temperature, amplitude, precipitation, relative humidity, sunlight and solar radiation. At harvest was evaluated the agronomic performance. In São Joaquim the cycle of Rebo is longer, the heat summation is lower, the precipitation and accumulated solar radiation are higher than in San Michele All'Adige. Lower temperatures and lower sunlight during flowering-veraison are the main climatic factors related to lower yields. In São Joaquim, lower temperatures and higher rates of solar radiation during maturation contribute positively in the formation of phenolic compounds, but make difficult acids degradation of Rebo variety.

Index terms: Vitis vinifera L., global solar radiation, growing degree days, phenology.
\end{abstract}

${ }_{1}^{1}$ (Trabalho 093-14). Recebido em: 28-02-2014. Aceito para publicação em; 14-05-2014.

${ }^{2}$ Eng. Agrônomo, Dr. Pesquisador da Epagri - Estação Experimental de São Joaquim. São Joaquim-SC. E-mail: albertobrighenti@epagri. sc.gov.br

${ }^{3}$ Eng ${ }^{a}$. Agrônoma, Dra. Bolsista PNPD-Capes, UFSC-Epagri. Florianópolis-SC, CEP 88034-901. E-mail: lucianeisabel@yahoo.com.br ${ }^{4}$ Eng. Agrônomo, Ph.D. Pesquisador Istituto Agrario di San Michele all'Adige - Fondazione Edmund Mach (IASMA - FEM), Via E. Mach, 138010, San Michele all'Adige, TN, Itália. E-mail: marco.stefanini@fmach.it

${ }^{5}$ Eng. Agrônomo, Ph.D. Pesquisador da Epagri-Ciram. Florianópolis-SC. E-mail: vieira@epagri.sc.gov.br

${ }^{6}$ Eng. Agrônomo, Ph.D. Professor, Universidade Federal de Santa Catarina, Centro de Ciências Agrárias, Departamento de Fitotecnia. Florianópolis-SC.E-mail: alsilva@cca.ufsc.br 


\section{INTRODUÇÃO}

A fenologia da videira apresenta forte relação com os fatores ambientais. Entre esses fatores, estão: a temperatura e a umidade do ar, a precipitação pluviométrica e a radiação solar. A interação destes fatores com o meio ambiente, assim como a variedade e as técnicas de cultivo são responsáveis pela potencialidade de cada região, bem como pela produtividade e qualidade das uvas (BOCK et al., 2011).

O potencial climático das regiões de elevada altitude para a produção de uvas viníferas (Vitis vinifera L.) em Santa Catarina, vem sendo comprovado através de várias pesquisas. Essas condições proporcionam clima diferenciado em relação às demais regiões vitícolas do Brasil, interferindo principalmente no ciclo fenológico. Devido às temperaturas do ar mais amenas nessas regiões, o ciclo vegetativo e reprodutivo da videira é mais extenso, acarretando maturação das uvas mais lenta para a produção de vinhos finos de qualidade (GRIS et al., 2010; VIEIRA et al., 2011; MALINOVSKI et al., 2012). Além disso, provoca a maturação em meses de menor frequência (abril e maio) e quantidade de precipitação pluviométrica, propiciando uvas com maior sanidade e qualidade enológica.

Nesse sentido, para determinar a potencialidade de novas variedades de videira em uma região, são importantes estudos e pesquisas sobre características edafoclimáticas e adaptação das variedades através de estudos de fenologia, maturação das uvas e interação entre esses fatores (HUNTER; BONNARDOT, 2011).

Com o objetivo de caracterizar as regiões de elevada altitude de Santa Catarina e de explicar a adaptação da variedade Rebo (Vitis vinifera L.) a essas condições específicas, foi realizado o presente trabalho com o intuito de comparar diferentes parâmetros climáticos em São Joaquim - SC, com San Michele All'Adige - TN, no norte da Itália.

\section{MATERIAL E MÉTODOS}

Para os estudos comparativos entre Brasil e Itália, utilizou-se dos dados fenológicos e meteorológicos da Estação Experimental de São Joaquim - Epagri (28¹6’30,08'S, 4956'09,34'O, altitude $1.400 \mathrm{~m}$ ), localizada em São Joaquim Santa Catarina. No estudo realizado na Itália, foram utilizados os dados do Instituto Agrário de San Michele All'Adige - Fondazione Edmund Mach (46 ${ }^{\circ} 11^{\prime} 41,46^{\prime \prime} \mathrm{N}, 11^{\circ} 08^{\prime} 04,41^{\prime \prime} \mathrm{L}$, altitude $\left.223 \mathrm{~m}\right)$, localizado em San Michele All'Adige, Província de Trento.

Em São Joaquim, o vinhedo foi implantado em 2006, com espaçamento de 3,0 m entre linhas e 1,5 m entre plantas, e sistema de condução tipo espaldeira. Em San Michele All'Adige, o vinhedo foi implantado no espaçamento de 3,0 m entre linhas, 1,0 $m$ entre plantas e sistema de condução tipo espaldeira (MATTIVI et al., 2000).

Em ambos os locais, utilizou-se dos dados fenológicos da variedade Rebo, que possui potencial para a produção de uvas e vinhos finos em Santa Catarina. Tal variedade foi obtida nos anos 20, pelo melhorista Rebo Rigotti, no programa de melhoramento da Estação Experimental Agrária de San Michele all'Adige, através do cruzamento entre as variedades Merlot x Teroldego e selecionado o "seedling" 107-3 pela sua constância de produção, resistência às doenças e pelas boas características produtivas e qualitativas (MATTIVI et al., 2000).

Neste trabalho, foram utilizadas as datas médias de ocorrência dos principais estádios fenológicos da variedade Rebo nos ciclos de 2010/2011, 2011/2012 e 2012/2013, em ambos os países estudados. Para comparar a fenologia de ambos os países, foram utilizados o número de dias após o solstício de inverno até a data de cada estádio fenológico. O início de brotação, a plena floração, a mudança de cor das bagas e a maturidade foram determinados segundo a classificação proposta por Baillod e Baggiolini (1993).

Os parâmetros climáticos foram avaliados segundo OMM (Organização Meteorológica Mundial), incluindo: temperaturas do ar média, máxima, mínima $\left({ }^{\circ} \mathrm{C}\right)$, amplitude térmica $\left({ }^{\circ} \mathrm{C}\right)$, precipitação pluviométrica $(\mathrm{mm})$, umidade relativa do ar média (\%), insolação (horas) e radiação solar global $\left(\mathrm{MJ} \mathrm{m}^{-2}\right)$. Para as avaliações deste trabalho, foram utilizados dados coletados ao longo de 30 anos em ambos os países.

Para a comparação climática de cada local, foi calculado o índice bioclimático vitícola em grausdias acumulados (GDA). Para o cálculo, considerouse a temperatura- base de $10^{\circ} \mathrm{C}$ (JONES et al., 2010), de acordo com a equação:

$\mathrm{GDA}=\Sigma$ máximo $\{[($ Tmáxima + Tmínima $) / 2]-10,0\}$;

Através do mosto, obtido com o esmagamento das bagas, foram determinados os Sólidos Solúveis Totais ( ${ }^{\circ}$ Brix), a Acidez Total Titulável (meq L $\left.{ }^{-1}\right)$ e o pH (OIV, 2009). A quantificação das antocianinas foi realizada através do $\mathrm{pH}$ diferencial, e o conteúdo de polifenóis totais foi determinado através do método de 
Folin-Ciocalteu, com leituras no espectrofotômetro. A produção por planta $\left(\mathrm{kg} \mathrm{planta}^{-1}\right)$ foi calculada levando-se em conta a massa fresca dos cachos e o número de cachos por planta.

Os resultados foram analisados através da estatística descritiva, e os dados do desempenho agronômico da variedade em São Joaquim correspondem à média dos ciclos 2010/2011, 2011/2012 e 2012/2013. Já os dados de San Michele All'Adige foram obtidos por Mattivi et al. (2000) entre os anos de 1980 e 1984.

\section{RESULTADOS E DISCUSSÃO}

A brotação da variedade Rebo, em São Joaquim, ocorreu em 31 de agosto, 71 dias após o solstício de inverno. Em San Michele All'Adige, a brotação ocorreu em 24 de abril, 123 dias após o solstício de inverno (Tabela 1). Acredita-se que essa diferença de 52 dias entre um local e outro seja devida às baixas temperaturas e ao inverno mais rigoroso a que as plantas são submetidas no norte da Itália, pois a data de brotação de uma variedade é determinada pelo somatório térmico (graus-dia acumulados) durante o período de ecodormência (BEGUM et al., 2007). Em San Michele All'Adige, as temperaturas médias dos meses de fevereiro e março foram $3,5^{\circ} \mathrm{C}$ e $8,6^{\circ} \mathrm{C}$, enquanto em São Joaquim, as temperaturas dos meses de julho e agosto foram $9,2^{\circ} \mathrm{C}$ e $10,9^{\circ} \mathrm{C}$, respectivamente.

A diferença entre os locais com relação à maturidade foi diferente; em São Joaquim, a maturidade ocorreu 294 dias após o solstício de inverno (11 de abril), enquanto em San Michele All'Adige a maturidade ocorreu 273 dias após o solstício de inverno (21 de setembro) (Tabela 1). A diferença na data da maturidade entre um local e outro foi de 21 dias. Acredita-se que essa aceleração no ciclo das plantas cultivadas na Itália tenha ocorrido devido à ocorrência de temperaturas mais elevadas na primavera e no verão italiano (Figura 1). A fenologia das plantas é uma resposta à duração dos dias, ou fotoperíodo, que por sua vez é regulado pela temperatura (WOLFE et al., 2005). Em San Michele All'Adige, a temperatura média desse período foi de $19,8^{\circ} \mathrm{C}$, enquanto em São Joaquim foi $16,1^{\circ} \mathrm{C}$ (Figura 1C).

O ciclo das plantas em São Joaquim foi mais longo do que em San Michele All'Adige em todos os subperíodos avaliados (Tabela 1). No subperíodo brotação-floração, o ciclo foi 42 dias mais longo, na floração-mudança de cor das bagas foi 19 dias mais longo e durante mudança de cor das bagasmaturidade foi 9 dias mais longo. No intervalo entre a brotação e a maturidade, o ciclo das plantas foi 69 dias mais longo.

Ao se observar o acúmulo térmico, a situação se inverte, em San Michele All'Adige foram registradas as maiores somas térmicas em todos os subperíodos avaliados, exceto entre as fases brotação-floração, cujo acúmulo térmico de São Joaquim foi superior (Tabela 1). Ao final do período entre a brotação e a maturidade, foi observado, em San Michele All'Adige, um acúmulo térmico de 267,3 graus-dia superior a São Joaquim.

As temperaturas máximas, médias e mínimas são, em média, 20 a 25\% superiores em San Michele All'Adige nos 5 subperíodos fenológicos avaliados (Figura 1). Esse fato justifica período mais curto para San Michele All'Adige e o prolongamento do ciclo da videira observado nas regiões de elevada altitude de Santa Catarina, quando comparadas com outros locais da Europa (VIEIRA et al., 2011). Em geral, temperaturas mais elevadas aceleram o desenvolvimento da planta e avançam a fenologia da videira (WOLFE et al., 2005).

A amplitude térmica média dos cinco subperíodos fenológicos avaliados em San Michele All'Adige foi de $12,4^{\circ} \mathrm{C}$, e de $9,3^{\circ} \mathrm{C}$ em São Joaquim (Figura 1). Tais valores podem ser considerados adequados, visto que uma amplitude térmica próxima a $10^{\circ} \mathrm{C}$ é ideal para produção de uvas de qualidade (JACKSON, 2008).

A umidade relativa foi, em média, 25\% superior em São Joaquim para todos os subperíodos avaliados (Figuras 1). A precipitação pluviométrica média observada ao longo do ciclo da videira, em São Joaquim, foi quase o triplo da de San Michele All'Adige, com valores de 1.214,6 mm e 453,4 $\mathrm{mm}$. Sem dúvida, as elevadas taxas de precipitação pluviométrica são um dos maiores limitantes da cultura da videira na região Sul do Brasil, visto que a frequência e a distribuição de chuvas são elementos climáticos de grande importância neste processo, porque constituem o fator primário principal para desencadear o início das infecções fúngicas na videira (CHAVARRIA et al., 2007).

A intensidade da radiação solar global acumulada $\left(\mathrm{MJ} \mathrm{m}^{-2}\right)$ foi mais elevada em São Joaquim em todos os subperíodos avaliados (Figura 1). No subperíodo brotação-floração, foi 73,8 \% mais elevada (Figura 1A), 3\% no subperíodo floraçãomudança de cor das bagas (Figura 1B) e 27\% mais elevada entre mudança de cor das bagas-maturidade e brotação-maturidade (Figura 1C e 1D). Nas regiões de altitude, os períodos de maior disponibilidade de radiação solar correspondem ao subperíodo brotação-floração. Resultados semelhantes foram 
obtidos por Campos et al. (2013), que observaram maior disponibilidade de radiação solar no final da primavera e início do verão.

As maiores diferenças na insolação foram observadas no subperíodo brotação-floração, sendo em São Joaquim registrados valores em média 40\% maiores do que em San Michele All'Adige (Figura 1A). No subperíodo seguinte de floração-mudança de cor das bagas, ocorreu o inverso e foram observados valores médios em torno de $20 \%$ inferiores em São Joaquim (Figura 1B). Nos demais subperíodos, os valores de insolação foram muito próximos nos dois locais avaliados. Este fato explica-se em parte pelo maior fotoperíodo em San Michele All'Adige que é resultante de sua maior latitude.

Os valores médios diários de radiação solar global, durante todo o ciclo, são mais elevados em San Michele All'Adige (19,4 $\mathrm{MJ} \mathrm{m}^{-2}$ ) do que em São Joaquim (16,9 $\left.\mathrm{MJ} \mathrm{m}^{-2}\right)$. Contudo, a radiação acumulada (Figura 2) foi maior em São Joaquim (3.711,6 $\mathrm{MJ} \mathrm{m}^{-2}$ ) do que em San Michele All'Adige (2.907,2 $\mathrm{MJ} \mathrm{m}^{-2}$ ), resultados estes que são provenientes de um ciclo de 69 dias mais longo em São Joaquim. Situação semelhante foi observada por Vieira et al. (2011), que registraram valores mais elevados de radiação global no planalto catarinense do que em Pech Rouge, na França.

Em San Michele All'Adige, as plantas produziram mais cachos, e a produtividade foi mais elevada (Tabela 1). As menores produtividades das plantas observadas em São Joaquim podem estar associadas com a menor insolação e a menor temperatura no subperíodo floração-mudança de cor das bagas (Figura 1B) que em San Michele All'Adige.

Afinal, fatores climáticos, como a entrada de luz solar no interior da copa e altas temperaturas $\left(>20^{\circ} \mathrm{C} \mathrm{e}<35^{\circ} \mathrm{C}\right.$ ), estão relacionados com o aumento da fertilidade das gemas, a qual é a capacidade de diferenciação de gemas vegetativas em frutíferas, e determinará sua produtividade (SÁNCHEZ; DOKOOZLIAN, 2005).

De acordo com diversos autores, o período a partir de 6 semanas após a brotação até a fase de mudança de cor das bagas é crítico, nesse intervalo ocorrem a indução e a diferenciação dos primórdios florais da videira que serão responsáveis pela produção de uvas no ciclo seguinte (HEAZLEWOOD; WILSON, 2004; WATT et al., 2008). Em São Joaquim, para a variedade Rebo, 6 semanas após a plena florada até a mudança de cor das bagas correspondem, em média, ao período entre o início de outubro e a primeira quinzena de fevereiro.

A ocorrência de temperaturas mais baixas do que aquelas registradas na Itália torna a produção de uvas em São Joaquim mais sensível a variações na produtividade causadas por condições ambientais (WATT et al., 2008). Essa situação pode ser comprovada pelos dados presentes na Tabela 1 , que comparou os índices produtivos da variedade Rebo no norte da Itália e no Sul do Brasil.

Na Tabela 1, podem ser observados os dados referentes à maturação tecnológica e à fenólica da variedade Rebo. No momento da colheita, foram observados teores de sólidos solúveis totais em torno de $20^{\circ}$ Brix em ambos os locais.

Porém, a variedade Rebo apresentou teores mais elevados de acidez total titulável e o pH ligeiramente mais baixo quando cultivada em São Joaquim (Tabela 1). A temperatura mais elevada é responsável pela atenuação do ácido málico e da acidez do mosto. Quando a uva amadurece em temperaturas mais baixas, as concentrações de ácido málico são sempre mais altas (PEREIRA et al., 2006).

Em São Joaquim, a concentração de antocianinas totais foi praticamente duas vezes maior que em San Michele All'Adige, acredita-se que, indiretamente, a altitude possa estar relacionada com esse resultado, já que Mateus et al. (2001) observaram um aumento nos teores de antocianinas das uvas à medida que a altitude aumentava. No entanto, é pouco provável que estes resultados sejam estritamente efeitos da altitude, mas sim os efeitos de diferentes condições climáticas de cada local. Os efeitos climáticos predominantes são a radiação solar e a temperatura (DOWNEY et al., 2006). Neste caso, as causas desta diferença podem estar relacionadas com a quantidade e a qualidade da radiação solar e com o termoperiodismo, que são efeitos da altitude.

A incidência da radiação solar nos cachos de uva também contribui para ativar o metabolismo e a formação de substâncias responsáveis pela qualidade do vinho, como os compostos fenólicos, especialmente os flavonóis (DOWNEY et al., 2006). Essa diferença pode conferir aos vinhos do planalto catarinense uma coloração mais intensa, com aroma mais complexo, maior estrutura e persistência (GRIS et al., 2010; MALINOVSKI et al., 2012).

As condições climáticas de São Joaquim favoreceram a maturação fenólica da uva, porque o acúmulo de antocianinas apresenta correlação negativa com as altas temperaturas e positiva com as baixas temperaturas durante a maturação. As uvas apresentam boa coloração quando a amplitude térmica não ultrapassa $10^{\circ} \mathrm{C}$, e durante a maturação deve ser inferior a $15^{\circ} \mathrm{C}$ (MORI et al., 2005).

Acredita-se que os teores de polifenóis totais ligeiramente mais elevados em San Michele All'Adige possam estar relacionados com as temperaturas mais altas registradas ao longo do ciclo. 
TABELA 1- Datas de ocorrência, duração cronológica média (dias) e disponibilidade térmica média (grausdia) dos principais estádios fenológicos, duração cronológica média (dias) e desempenho agronômico da variedade Rebo em São Joaquim-SC e em San Michele All'Adige-TN, nos ciclos 2010/2011, 2011/2012, 2012/2013.

\begin{tabular}{|c|c|c|}
\hline \multirow{3}{*}{ Estádio Fenológico } & \multicolumn{2}{|c|}{ Local } \\
\hline & São Joaquim & San Michele All'Adige \\
\hline & $\left(28^{\circ} 16 ’ 30,08 ’ \mathrm{~S}, 4^{\circ} 56^{\prime} 09,344^{\prime} \mathrm{O}\right)$ & $\left(46^{\circ} 11^{\prime} 41,46^{\prime \prime} \mathrm{N}, 1^{\circ} 08^{\prime} 04,41^{\prime \prime} \mathrm{L}\right)$ \\
\hline Início Brotação & 31-ago & $24-a b r$ \\
\hline Plena Florada & 24-nov & 2-jun \\
\hline Mudança Cor Bagas (50\%) & 14-fev & 10-ago \\
\hline Maturidade & $11-\mathrm{abr}$ & 21-set \\
\hline \multicolumn{3}{|c|}{ Estádio Fenológico (dias após o solstício de inverno) } \\
\hline Início da Brotação & 71 & 123 \\
\hline Plena Florada & 156 & 162 \\
\hline Mudança Cor Bagas (50\%) & 238 & 231 \\
\hline Maturidade & 294 & 273 \\
\hline \multicolumn{3}{|l|}{ Duração Cronológica Média (dias) } \\
\hline Brotação-Floração & 81 & 39 \\
\hline Floração-Mud. Cor Bagas & 88 & 69 \\
\hline Mud. Cor Bagas-Maturidade & 51 & 42 \\
\hline Brotação-Mud. Cor Bagas & 169 & 108 \\
\hline Brotação-Maturidade & 219 & 150 \\
\hline \multicolumn{3}{|c|}{ Disponibilidade Térmica Média (graus dia) } \\
\hline Brotação-Floração & 312,4 & 290,9 \\
\hline Floração-Mud. Cor Bagas & 683,7 & 866,7 \\
\hline Mud. Cor Bagas-Maturidade & 335,1 & 440,9 \\
\hline Brotação-Mud. Cor Bagas & 996,1 & $1.157,6$ \\
\hline Brotação-Maturidade & $1.331,2$ & $1.598,5$ \\
\hline \multicolumn{3}{|l|}{ Desempenho Agronômico } \\
\hline $\mathbf{N}^{\circ}$ Cachos & 17,1 & $32,8 *$ \\
\hline Produtividade (kg planta $\left.{ }^{-1}\right)$ & 1,3 & $7,1 *$ \\
\hline Sólidos Solúveis Totais ( ${ }^{\circ}$ Brix) & 20,2 & $20,2 *$ \\
\hline Acidez Total Titulável (Meq L L-1) & 173,3 & $102,6 *$ \\
\hline pH & 3,2 & $3,0 *$ \\
\hline Antocianinas Totais & $1.143,2$ & $621,0 *$ \\
\hline Polifenóis Totais & $1.298,4$ & $1.360,0 *$ \\
\hline
\end{tabular}

\footnotetext{
* Fonte: Mattivi et al.(2000)
} 


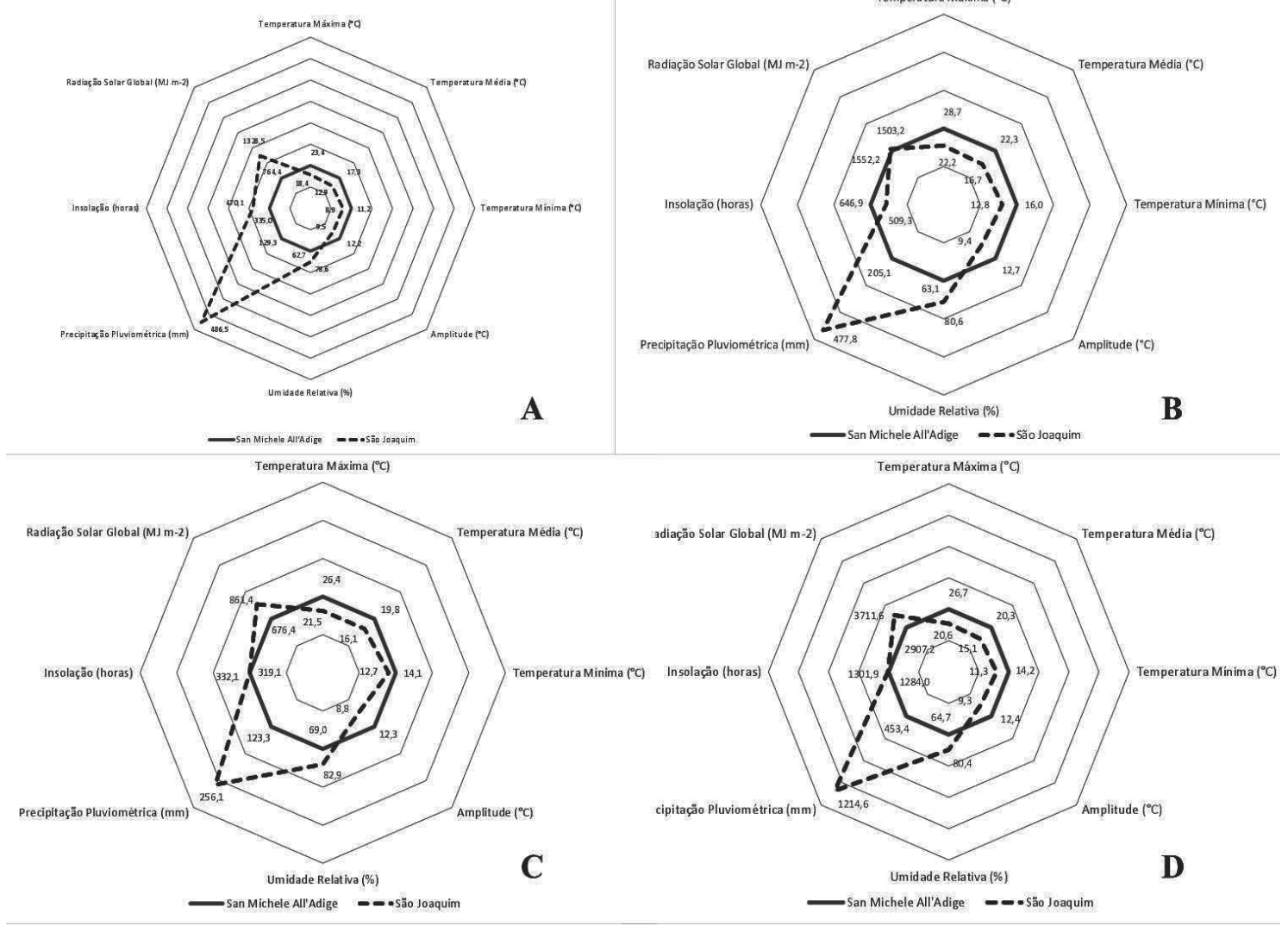

FIGURA 1- Parâmetros climáticos observados em São Joaquim e San Michele All'Adige nos subperíodos brotação-floração (A), floração-mudança de cor das bagas (B), mudança de cor das bagasmaturidade (C) e brotação-maturidade (D) da variedade Rebo. Tendo como base percentual os valores de San Michele All'Adige para cada variável climática. Média dos ciclos 2010/2011, 2011/2012 e 2012/2013.

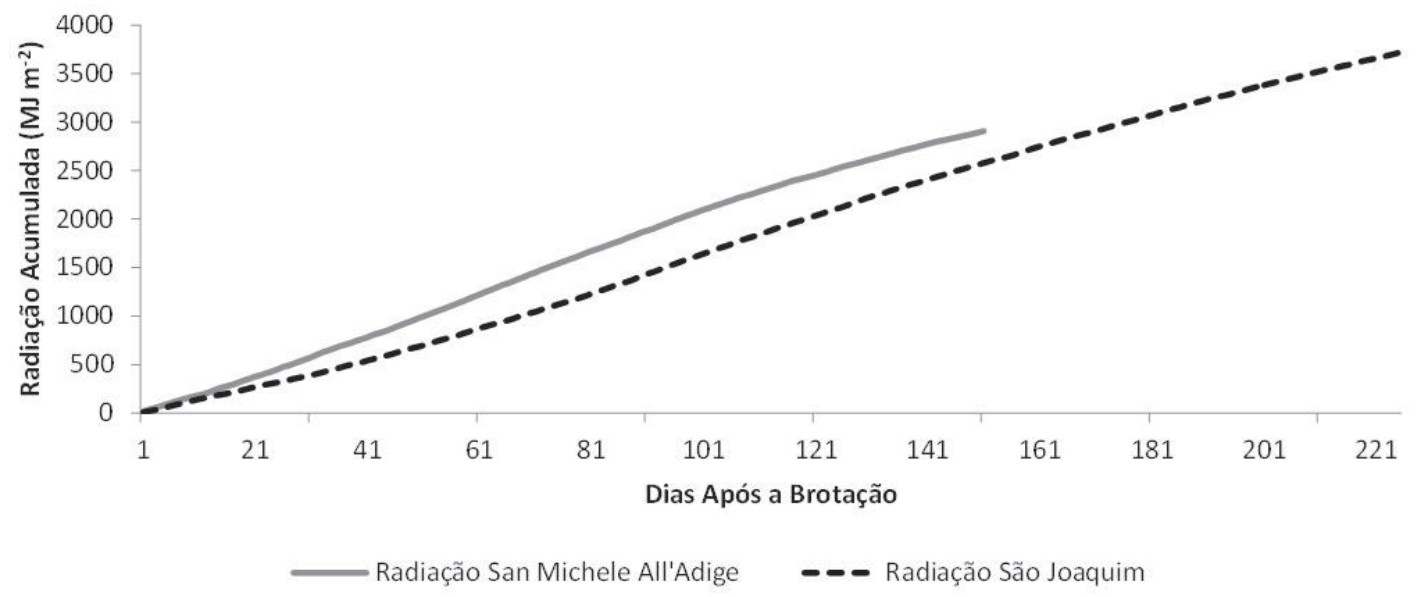

FIGURA 2 - Radiação solar global acumulada no subperíodo brotação-maturidade medida em São Joaquim e San Michele All'Adige. Média de um período de 30 anos. 


\section{CONCLUSÕES}

Devido às temperaturas amenas em São Joaquim, o ciclo da variedade Rebo é mais longo, e o acúmulo térmico é menor. As principais diferenças observadas nos parâmetros climáticos dos locais dizem respeito às elevadas taxas de precipitação pluviométrica e ao maior acúmulo de radiação solar global, ocasionado pela maior duração do ciclo em São Joaquim.

Acredita-se que temperaturas mais amenas e a menor insolação durante o subperíodo floração-mudança de cor das bagas (que coincide com a indução e diferenciação do primórdio floral) sejam os principais fatores climáticos relacionados com as menores produtividades das plantas em São Joaquim.

A elevada taxa e frequência de precipitação pluviométrica é o principal fator climático limitante da cultura da videira nas regiões de altitude elevada do Sul do Brasil, em comparação com San Michele All'Adige.

Durante o período de maturação, as baixas temperaturas e o elevado acúmulo de radiação solar global são os principais fatores climáticos que diferenciam as regiões avaliadas e interferem de forma marcante na qualidade da uva. Em São Joaquim, tais condições favorecem o acúmulo de polifenóis, particularmente a síntese de antocianinas, mas dificultam a degradação dos ácidos.

\section{REFERÊNCIAS}

BAILLOD, M.; BAGGIOLLINI, M. Les stades repères de la vigne. Revue Suisse Viticulture, Arboriculture, Horticulture, Lausanne, v. 25, n. 1, p. 7-9, 1993.

BEGUM, S.; NAKABA, S.; ORIBE, Y.; KUBO, T.; FUNADA, R. Induction of cambial reactivation by localized heating in a deciduous hardwood hybrid poplar (Populus sieboldii x P. grandidentata). Annals of Botany, London, v.100, p.439-447, 2007.

BOCK, A.; SPARKS, T.; ESTRELLA, N.; MENZEL, A. Changes in the phenology and composition of wine from Franconia, Germany. Climate Research, Amelinghausen, v. 50, p. 69-81, 2011.
CAMPOS, C.G.C.; VIEIRA, H.J.; BACK, A.J.; SILVA, A.L. Fluxos de radiação solar global em vinhedos de altitude de São Joaquim-SC. Revista Brasileira de Fruticultura, Jaboticabal, v.35, n.3, p.722-729, 2013.

CHAVARRIA, G.; SANTOS, H.P.; SÔNEGO, O.R.; MARODIN, G.A.B.; BERGAMASCHI, H.; CARDOSO, L.S. Incidência de doenças e necessidade de controle em cultivo protegido de videira. Revista Brasileira de Fruticultura, Jaboticabal, v.29, n.3, p.477-482, 2007.

DOWNEY, M.O.; DOKOOZLIAN, N.K.; KRSTIC, M.P. Cultural practice and environmental impacts on the flavonoid composition of grapes and wine: a review of recent research. American Journal of Enology and Viticulture, Davis, v.57, p. 257-268, 2006.

GRIS, E.F.; BURIN, V.M.; BRIGHENTI, E.; VIEIRA, H.J. ; BORDIGNON-LUIZ, M. Phenology and ripening of Vitis vinifera grape varieties in São Joaquim, southern Brazil: a new South American wine growing region. Ciência e Investigación Agraria, Santiago, v. 37, p. 61-75, 2010.

HEAZLEWOOD, J.E.; WILSON, S. Anthesis, pollination and fruitset in Pinot Noir. Vitis, Siebeldingen, v. 43, p. 65-68, 2004.

HUNTER, J.J.; BONNARDOT, V. Suitability of Some Climatic Parameters for Grapevine Cultivation in South Africa, with Focus on Key Physiological Processes South African Journal of Enology and Viticulture, Davis, v. 32, n. 1, p.137-154, 2011.

JACKSON, R.S. Wine science: principles and applications. $3^{\text {rd }}$ ed. São Diego: Elsevier, 2008. 789 p.

JONES, G.; DUFF, A.; HALL, A.A.; MYERS, J.W. Spatial analysis of climate in winegrape growing regions in the Western United States. American Journal of Enology and Viticulture, Davis, v.61, p. 313-326, 2010.

MALINOVSKI, L.I.; WELTER, L.J.; BRIGHENTI, A.F.; VIEIRA, H.J; GUERRA, M.P.; DA SILVA, A.L. Highlands of Santa Catarina/Brazil: A region with high potential for wine production. Acta Horticulturae, The Hague, v. 931, p.433-440, 2012. 
MATEUS, N. ; MARQUES, S. ; GONÇALVES, A.C. ; MACHADO, J.M. ; DE FREITAS, V. Proanthocyanidin composition of red Vitis vinifera varieties from the douro valley during ripening: influence of cultivation altitude. American Journal of Enology and Viticulture, Davis, v.52, p.115-121, 2001.

MATTIVI, F.; MALOSSINI, U.; RONCADOR, I.; NICOLINI, G. Characterization of polyphenols of Rebo (IR 107-3) wines in comparison with other Rigotti crosses and related varieties. Symposium on Grapevine Genetics and Breeding, Acta Horticulture, The Hague, v. 528, p.693-699, 2000.

MORI, K.; SUGAYA, S.; GEMMA, H. Decreased anthocyanin in grape berries grown under elevated night temperature condition. Scientia Horticulturae, Amsterdam, v.105, n.3, p. 319-330, 2005.

OIV - Office International de la Vigne et du Vin. Recueil des méthodes internationales d'analyse des vins et des moûts. Paris, 2009. 368p.

PEREIRA, G.E.; GAUDILLÈRE, J.P.; PIERI, P.; HILBERT, G.; MAUCOURT, M.; DEBORDE, C. Microclimate influence on mineral and metabolic profiles of grape berries. Journal of Agricultural and Food Chemistry, Easton, v.54, p.6765-6775, 2006.
SÁNCHEZ, L.A.; DOKOOZLIAN, N.K. Bud microclimate and fruitfulness in Vitis vinifera L. American Journal of Enology and Viticulture, Davis, v.56, p. 319-329, 2005.

VIEIRA, H.J.; BACK, A.J.; SILVA, A.L.; PEREIRA. E.S. Comparação da disponibilidade de radiação solar global e fotoperíodo entre as regiões vinícolas de Campo Belo do Sul-SC, Brasil e Pech Rouge, França. Revista Brasileira de Fruticultura, Jaboticabal, v. 33, n. 4, p.1055-1065, 2011.

WATT, A.M.; DUNN, G.M.; MAY, P.B.; CRAWFORD, S.A.; BARLOW, E.W.R. Development of inflorescence primordia in Vitis vinifera L. cv. Chardonnay from hot and cold climates. Australian Journal of Grape and Wine Research, Glen Osmond, v.14, p.46-53, 2008.

WOLFE, D.W.; SCHWARTZ, M.D.; LAKSO, A.N.; OTSUKI, Y.; POOL, R.M.; SHAULIS, N.J. Climate change and shifts in spring phenology of three horticultural woody perennials in northeastern USA. International Journal of Biometeorology, Heidelberg, v.49, p.303-309, 2005. 